\title{
Corrigendum: Effectiveness of Physical Activity Intervention on ADHD Symptoms: A Systematic Review and Meta-Analysis
}

\author{
Yongtao Xie ${ }^{1,2+}$, Xuping Gao ${ }^{3+}$, Yiling Song ${ }^{1}$, Xiaotong Zhu ${ }^{1}$, Mengge Chen ${ }^{1}$, Li Yang ${ }^{3 *}$ and \\ Yuanchun Ren ${ }^{1 *}$ \\ ${ }^{1}$ College of Physical Education and Sports, Beijing Normal University, Beijing, China, ${ }^{2}$ Department of Human Movement \\ Science, Hebei Sports University, Shijiazhuang, China, ${ }^{3}$ Department of Child \& Adolescent Psychiatry, National Clinical \\ Research Center for Mental Disorders and NHC Key Laboratory of Mental Health (Peking University Sixth Hospital), Peking \\ University Sixth Hospital (Institute of Mental Health), Beijing, China
}

Keywords: attention-deficit/hyperactivity disorder, physical activity, intervention, motor skill, effectiveness

\section{OPEN ACCESS}

Approved by:

Frontiers Editorial Office,

Frontiers Media SA, Switzerland

*Correspondence:

Yuanchun Ren

yuanchun-ren@bnu.edu.cn

Li Yang

yangli_pkuimh@bjmu.edu.cn

${ }^{\dagger}$ These authors have contributed equally to this work and share first authorship

Specialty section

This article was submitted to Child and Adolescent Psychiatry,

a section of the journal

Frontiers in Psychiatry

Received: 31 October 2021 Accepted: 10 November 2021 Published: 06 December 2021

Citation:

Xie Y, Gao X, Song Y, Zhu X, Chen M,

Yang $L$ and Ren $Y$ (2021)

Corrigendum: Effectiveness of Physical Activity Intervention on ADHD Symptoms: A Systematic Review and Meta-Analysis.

Front. Psychiatry 12:806241. doi: 10.3389/fpsyt.2021.806241

\section{A Corrigendum on}

Effectiveness of Physical Activity Intervention on ADHD Symptoms: A Systematic Review and Meta-Analysis

by Xie, Y., Gao, X., Song, Y., Zhu, X., Chen, M., Yang, L., and Ren, Y. (2021). Front. Psychiatry 12:706625. doi: 10.3389/fpsyt.2021.706625

In the published article, there was an error in the order of the authors in the correspondence section. Yuanchun Ren should be listed before Li Yang.

In the original article, $3 \mathrm{rd}$ sentence of the 2 nd paragraph in discussion section should be changed from "Frederiksen et al. reviewed eight articles and suggested that PA was more beneficial for anxiety populations compared to cognitive behavioral therapy (60)." to "Frederiksen et al. reviewed eight articles and suggested PA add-on was more beneficial for anxiety populations compared to cognitive behavioral therapy only (60)."

The authors apologize for this error and state that this does not change the scientific conclusions of the article in any way. The original article has been updated.

\section{REFERENCES}

60. Frederiksen KP, Stavestrand SH, Venemyr SK, Sirevåg K, Hovland A. Physical exercise as an add-on treatment to cognitive behavioural therapy for anxiety: a systematic review. Behav Cogn Psychother. (2021) 49:626-40. doi: $10.1017 / S 1352465821000126$

Publisher's Note: All claims expressed in this article are solely those of the authors and do not necessarily represent those of their affiliated organizations, or those of the publisher, the editors and the reviewers. Any product that may be evaluated in this article, or claim that may be made by its manufacturer, is not guaranteed or endorsed by the publisher.

Copyright (c) 2021 Xie, Gao, Song, Zhu, Chen, Yang and Ren. This is an open-access article distributed under the terms of the Creative Commons Attribution License (CC BY). The use, distribution or reproduction in other forums is permitted, provided the original author(s) and the copyright owner(s) are credited and that the original publication in this journal is cited, in accordance with accepted academic practice. No use, distribution or reproduction is permitted which does not comply with these terms. 